\title{
DIY Design Process for Interactive Surfaces
}

\author{
Jennifer G. Sheridan ${ }^{1}$, James Tompkin², Abel Maciel ${ }^{3}$, George Roussos ${ }^{1}$ \\ 1 London Knowledge Lab \\ 23-29 Emerald St. \\ London UK \\ +44 (0)20 77632137 \\ ${ }^{2}$ Department of Computer Science \\ ${ }^{3}$ Bartlett School of Architecture \\ University College, London UK \\ +44 (0)20 76790357 \\ j.tompkin@cs.ucl.ac.uk; a.maciel@ucl.ac.uk
}

j.sheridan@ioe.ac.uk; g.roussos@dcs.bbk.ac.uk

\begin{abstract}
This paper charts the design and build of two interactive tabletops that use infrared (IR) illumination techniques. One table implements fiducial tracking, whilst the other implements multi-touch tracking. Trade-offs in both designs are discussed to highlight key considerations when building an interactive table. Using three key dimensions from lessons learned, we conduct a comparative analysis of both approaches. Finally, we propose a DIY Design Process to assist designers in building their own interactive table.
\end{abstract}

\section{Categories and Subject Descriptors}

B.m [Hardware]: Miscellaneous. D.2.13 [Software]: Reusable Software - Reusable libraries. H5.2 [Information Systems]: Information interfaces and presentation (I.7) - User Interfaces (D2.2, H1.2, I.3.6). K.3 [Computing Milieux]: Computers in Education - Computer uses in education.

\section{General Terms}

Measurement, Documentation, Design, Reliability, Experimentation, Human Factors, Standardization.

\section{Keywords}

Tabletop, multi-touch, interactive surface, TUIO, fiducial, infrared, diffusion, design.

\section{INTRODUCTION}

Interactive tables come in many shapes and forms and are created for different interaction styles and concepts. Many examples of interactive tables exist (e.g. DigitalDesk [1], DiamondTouch [2], metaDESK [3], Playanywhere [4]) and recently, commercially available interactive tabletops have been introduced to the consumer, such as the Microsoft Surface, Philips Entertaible and NUI[5]. However, discussion about how these tables were designed has not been adequately disseminated. In fact, little has been reported in the literature about the different design decisions and trade-offs that are made when constructing interactive tables despite the fact that such decisions can have a profound impact on a successful outcome.

Moreover, the majority of interactive tables that are in use today are custom-made, meaning that there is no off-the-shelf solution or defined way to build a table - developers must make design solutions based on their unique requirements, skills and expertise. Typically, researchers and developers use a trial-anderror approach to building their table which can be both costly

(C) The Author 2009.

Published by the British Computer Society and time consuming and, in the case of research, divert attention and resources away from the main focus of the particular investigations.

To be sure, adopting a DIY approach to the design of interactive tables allows developers to gain a distinct advantage since they can customize the table in ways that are not possible with commercial technology. For example, in research we can link together different sensor devices, projection equipment, or sound in unconventional ways, which are not normally allowed with tightly controlled platforms - a limitation that has been recently highlighted by the severe restrictions placed on extending mobile phone hardware for example.

A core ingredient of many interactive tables is the use of infrared illumination (IR) to recognize objects or fingers that come in contact with the surface. reacTIVision [4] is one such system which provides a computer-vision framework that uses markers known as 'fiducials' to link physical objects in the real world with digital representations in the virtual world. reacTIVision uses the TUIO [5] protocol for transmitting the state of tangible objects and multi-touch control on a table surface. Other libraries, such as TouchLib, can be used to generate TUIO events to transform a table into a multi-touch interaction surface. IR illumination is used in combination with these libraries to improve marker or multi-touch recognition.

Recently, the reacTIVision framework has grown in popularity and is even being used in live performances by pop icons such as Björk [6]. This increasing popularity and use of interactive tables suggests a greater need for understanding the design trade-offs for building interactive tables.

Our paper describes the iterative design and development of two tables that use IR illumination and the TUIO library for tracking object and multi-touch interaction. Even though our tables were designed at different locations and with different studies in mind, we overcame many similar technical hurdles to implement our systems.

We begin with a description of our conceptual design, intended user group and environment. We discuss the design considerations and decisions made at various stages of the physical development, and then compare and discuss design tradeoffs and the lessons learned. We then use these lessons learned to conduct a comparative analysis. We conclude with a description of suggested improvements and guidelines for the development of IR illuminated interactive tables.

Our aim is to assist developers in making good design decisions when building interactive tables to help flatten the learning curve and reduce development time. Our paper acts as a stepping-stone for future research and development of interactive surfaces.

\section{BACKGROUND}

The tangible table is part of the Designing Tangibles for Learning project (DeTaLe) [7] at the London Knowledge Lab, which aims to systematically investigate and understand how different ways of linking together objects, environments and 


\section{J. Sheridan et al.}

information affect the way that learners interact with and understand scientific ideas [8]. Based on a research framework [9], the aim of the project is to identify the design characteristics of tangible artefact-action representation relationships (i.e., the different ways of linking information and representations of scientific phenomena to objects and actions placed upon those objects). We constructed an interactive table to allow children to explore scientific concepts whilst interacting with physical artefacts on the table surface.

We designed the multi-touch table at University College London to study collaborative group work; specifically, to investigate the effect of participant feedback. Our large multitouch table allowed four adults to collaboratively play a version of the board game Carcassonne [10]. Interactions mimicked the norms of the conventional board game whilst granting the benefits of the virtual form. The project lasted for three months, during which the table was developed on a limited budget and the user study was conducted. Many of the design decisions that we made during construction were based on a need to re-use existing equipment. However, these often conveniently matched the format and dimensional requirements of the study.

\section{CONCEPT DESIGN}

In designing the tangible table our first decision was to determine exactly which scientific concept we wanted to employ in our study. First, we talked with a Physics teacher to better appreciate the concepts students had difficultly in understanding and we settled on light comprehension (refraction, reflection, transmission). We then conducted a design workshop with various adults in our lab to help us to understand any misconceptions in comprehension as well as to develop paper prototypes of possible interaction scenarios. This helped us to uncover any shared misconceptions and we then used these misconceptions to design our interaction scenarios.

For the multi-touch table we took a different approach. Our intention was to study the decision making process in collaborative group work. Whilst we considered various tasks such as master plan design and office layout, we decided to use a board game for its simplicity and brevity. We chose the award-winning board game Carcassonne for many reasons: it is simple to learn; takes 30 minutes to play; and, generates complex behaviour fast [9]. Also, we chose this game for many design-related reasons: the game is 'flat' and can be displayed in 2D; participants can view and play from any angle around a tabletop; the game does not require any 'private' elements (e.g. players' hidden Scrabble letters or a Poker hand) which would complicate the use of a shared display; and finally, the main interaction in the game is placing tiles and counters. This interaction is easy to translate to a multi-touch table. Other game mechanics (such as the rolling of dice) are harder to accomplish satisfactorily in a virtual game.

\subsection{User group and environment}

Our user group for the tangible table was determined early on: children of various age groups. Since we were designing for children, our table had to be extremely safe, able to withstand potentially rough play, and our tangible artefacts had to be sized and weighted appropriately for a child's hands. Reflection and analysis of our user studies, highlighted in [12;13], provided insight into how to redesign and develop our table and artefacts so that they were suited to our target user group.

Studies undertaken with the tabletop involved twenty-one students, aged 11-12 years, working in groups of three. They had little or no previous formal knowledge of the physics of light. All students found the environment intuitive and easy to use, and highly enjoyable. Their perception of enjoyment was directly related to their perceived level of activity, which in this environment was highly dependent on action and manipulation.
Collectively the findings suggest a number of implications for tangible environments for learning: the value of explicit awareness of others actions in facilitating exploration, collaborative construction, and interpretation [14]; design issues around mappings of real-world objects to virtual environments, highlighting the potential impact on learning of mixed metaphors [14]; and, the potential of the environment for supporting collaboration through 'peer interference' in learning activities [15]. Furthermore, the teachers were very impressed by the application and thought it could be a powerful tool for use in classes.

The multi-touch table was designed for use by groups of four adults. We did not place restrictions on age, gender, occupation or group member familiarity. Each of the eleven groups was tasked with collaborating to solve a puzzle version of Carcassonne, whilst the actions of individual group members were tracked to provide real-time participation feedback. This feedback was to aid the groups in self-modulating their individual member's participation levels. Whilst this kind of system is broadly possible with other technologies and human assistance, the multi-touch table and hand tracking makes the experience fully automatic and largely transparent with no special considerations necessary from the participants.Both teams intended to develop a portable table - one that would be set up in a lab but also could be moved for on-site testing or public interaction. In both studies, the tables were developed in an indoor lab space, both of which had controllable ambient lighting.

\section{PHYSICAL DESIGN}

As the DeTaLe project focused on tangible interaction, we needed a system which could bind physical artefacts with digital representations. The reacTable [13] provides a robust example of an interactive table using a computer-vision framework. The framework, called reacTIVision [4], is open source, cross-platform and has been significantly documented and tested. reacTIVision tags, called 'fiducial' markers, bind physical artefacts with digital representations. Our intention was to build a tangible table based on the specifications outlined in [4], where each fiducial marker represented a light concept (e.g. reflection, refraction and transmission).

Like the tangible table, the multi-touch table used a visionbased diffusion illumination system. We chose this system as opposed to another multi-touch technique, such as capacitive or frustrated total internal reflection (FTIR), since our project timeline was short and diffusion illumination systems are quicker to build than other types of systems. Our intention was to build a multi-touch table using diffusion illumination to detect when users were moving game pieces around the surface with their fingers.

Designs were calculated differently. For the tangible table, all of the designs were drawn on paper whereas the multi-touch table was simulated using CAD software to work out how to achieve the required projection within set tolerances. This also allowed for $\mathrm{CNC}$ production of smaller parts such as a switch box.

\subsection{Table surface}

As discussed in [4], the table surface is the most important component of the system in that all activity will be carried out on the surface. In both cases, obtaining the proper material for the surface was very important in that it had to take considerable weight without flexing; diffuse infrared illumination for projection; and be robust to repeated use.

Both tables underwent an iterative design and prototyping process. The first prototype of the tangible table was made from a camping table, a paper picture frame and a low-lumens projector (Figure 1). The initial prototype of the multi-touch 
table set tracing paper on a poster frame balanced between two shelves (Figure 2).

The first priority was defining how large the surface needed to be to suit each study. Both tables intended to use rear-projection for the display area. The distance from projector to the table surface defines the possible size of the projected image. This distance determines the height of the table: a bigger image will require a bigger distance and thus a higher table. Often shortthrow projectors and mirrors are used to keep the table height low but produce a large projected image. Since the height of the tangible table was restricted to the average height of a schoolaged child, we purchased an ultra short-throw projector for producing a large image at a low height (Figure 1).

As the multi-touch table was intended for adult use, the height restriction was more flexible. The multi-touch designers followed Ryall's [14] suggestion that the surface should be at least $1070 \mathrm{~mm}$ large by the diagonal. The larger area can accommodate more people and helps reduce elbow knocking or 'personal area' problems. Since we did not have access to a short-throw projector, we needed a much larger distance to produce an image at least as large as $1070 \mathrm{~mm}$ diagonal. We used a mirror to increase the projection distance and in turn reduce the minimum height required for our table (Figure 2).

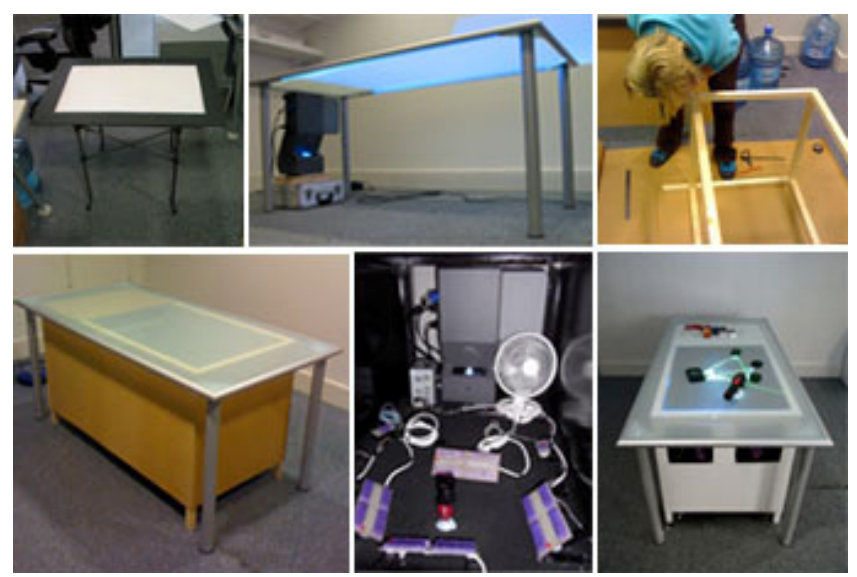

Figure 1. Iterative development of the tangible table. Paper frame and camping table (top left); Sanyo PLC projector and IKEA table (top middle); constructing the housing (top right); final prototype (bottom left); interior including camera and IR LEDs (bottom middle); final design running the light scenarios in Processing (bottom right).

In both studies, the table surface required diffusion material to show the projected image. To test different diffusion materials and surfaces for the tangible table we obtained several samples of Perspex of varying thickness, frosting and clarity, placed a fiducial on the frosted side and held this approximately $250 \mathrm{~mm}$ from our camera. We also tried using clear Perspex with tracing paper as described in [16]. We determined that we needed a surface that was less than $10 \mathrm{~mm}$ thick (to reduce refraction effects), was only frosted on one side, and did not contain any pattern. We purchased an IKEA VIKA LAURI tempered glass surface $(1570 \times 780 \mathrm{~mm})$ frosted on the underside with a maximum load of $50 \mathrm{~kg}$ in a lightweight aluminum steel frame and VIKA CURRY legs (700mm) (Figure 1).

The multi-touch table surface needed to be smooth enough for finger touches. We acquired a piece of polycarbonate, which had been used in a CAVE installation at UCL. This turned out to be a great advantage. The material was designed to be an excellent projection surface, was diffuse on one side and glossy on the other, and was $10 \mathrm{~mm}$ thick. Our final interactive surface measured $1040 \times 780 \mathrm{~mm}$ (roughly equal to the resolution of our projector and camera). Also, it is often convenient for the surface to be higher than the required height: for example, when using the surface as a work surface when standing [17]. We decided to raise the table to standing height to $(835 \mathrm{~mm})$ to provide ample legroom.

\subsection{Camera}

Both studies had to determine the best possible placement for the camera. The tangible tabletop was limited by its height restriction (Figure 1) and the multi-touch table was limited by its mirror placement (Figure 2).

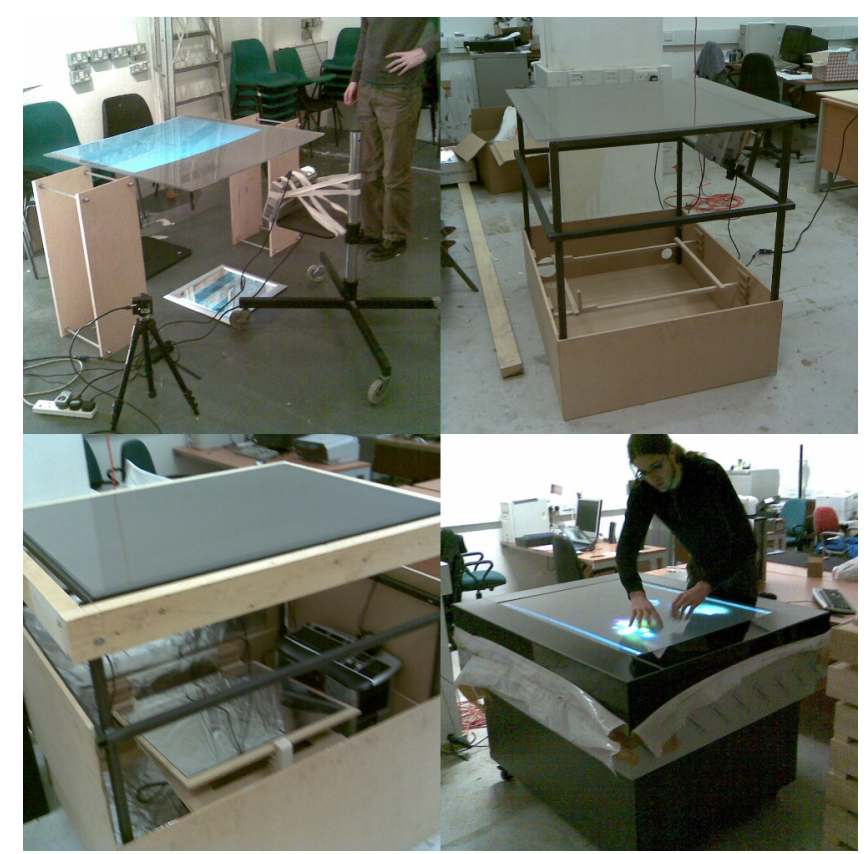

Figure 2: Clockwise from top right: initial experiment (camera not correctly placed); steel frame, surface material and projector/camera mount; mirror and housing (foil was not used in final version); testing the nearly complete table with wide bezel top.

The first camera tested for the tangible table was a Unibrain Fire-i camera $(30 \mathrm{~Hz})$ with a $4.3 \mathrm{~mm}$ lens. However, the Fire-i is a low-resolution $(640 \times 480)$ camera and in our case we could only track one fiducial at a time at a maximum focal length of $100 \mathrm{~mm}$. The addition of a $2.1 \mathrm{~mm}$ wide lens (no IR coating) allowed us to track more fiducials; however, we could not achieve the clear image that we needed beyond $100 \mathrm{~mm}$. Our second choice was an AVT Guppy F-080B Firewire $(30 \mathrm{~Hz}$, monochrome) camera. For the multi-touch table, we employed a Point Grey Dragonfly2 Firewire camera (XGA, 30Hz, monochrome).

Without wide-angle or fish-eye lenses, it is often hard for the camera to see the entire interaction surface, particularly in systems with little vertical height. To resolve this problem, we used a wide angle GOYO $1 / 2$ " F1.4/3.5mm manual iris CCTV lens, increased our resolution $(1032 \times 778)$ and distance $(300 \mathrm{~mm})$ for the tangible table and placed the camera under the middle of the interaction surface on the floor of the housing. We corrected barrel distortion using reacTIVision. In the multitouch system, we used a Clover L-BW3580SD Varifocal Auto Iris lens, placing the camera as close to the projector as possible to minimize parallax effects. The camera then viewed the table surface via a mirror (Figure 4). Due to the offset and slight barrel distortion, certain areas of the surface had more imaging 


\section{J. Sheridan et al.}

plane resolution than others, but this did not significantly affect performance.

Both the Guppy and Dragonfly2 came with imaging preprocessing software for controlling camera properties. The AVT Guppy software (only available in Windows) allowed us to control brightness (16), shutter (3093) and gain (232) through software. The Dragonfly2 software helped control shutter and exposure time. This greatly aided the tracking process.

\subsection{IR Filter}

Since both tables used IR illumination techniques for tracking, an IR camera lens or filter was needed to cut out any visible light. For the tangible table, we used the technique described by Antle [16], and used a loose visually opaque polyester technical filter with transmission over 730 nanometers. We obtained a sample package of $75 \times 75 \mathrm{~mm}$ filters from Lee Filters (leefilters.com) and using BluTack, we stuck the filter on top of our lens.

For the multi-touch table, the IR cutoff filter was removed from in front of the camera's CCD and replaced with a plastic IR longpass filter attached over the lens with brown packing tape. Ideally, an IR bandpass filter would be used (matched to the wavelength of the illumination) to reduce potential noise. However, bandpass filters are more expensive.

\subsection{Projection and mirror}

The majority of interactive tables, and indeed the reacTable itself, use a mirror to reflect the projector image onto the table surface and thus increase the size of the interaction surface. One of the more difficult aspects of building an interactive table is aligning the projector, camera and mirror. Most systems consist of an inexpensive (1500-2000 lumens) projector placed at an angle underneath the table surface, and mirror to reflect the image.

Recently, Sanyo introduced the PLC-XL50 - a short-throw projector with built in mirror (Figure 1). Although the PLCXL50 is moderately large $(374 \times 196.8 \times 495 \mathrm{~mm})$ and heavy $(16.74 \mathrm{~kg})$ compared to lower lumens projectors, its housing is robust and it is light enough for an adult to lift with one arm, thus making it portable. Most importantly, it eliminates the need for a mirror. When placed on its head for tabletop projection, the projector can be as close as $3 \mathrm{~cm}$ from the table surface with the projected image remaining in focus. This produces a very large projected image from an extremely short distance.

The multi-touch table also used a business projector (Hitachi CP-X275 XGA) as they are generally much brighter than home cinema projectors. As our projector and camera both operated at XGA resolutions, and our interaction surface was 1040x780mm, our table has a rough 1pixel:1mm precision. The projector's support frame was design for easy adjustment, sliding sideways to align projection with projection surface. Our mirror needed to be $600 \times 600 \mathrm{~mm}$ in size to sit at the bottom of our table and reflect the projected image. Ideally, a first-surface mirror would be used to remove refraction effects but these are expensive and fragile, and so we acquired a low-cost acrylic mirror, attaching it to an adjustable base so that we could change the angle of reflection.

\subsection{Housing}

Both studies constructed a housing unit for placing tracking and illumination equipment under the table. Since we had purchased a table for the tangible study, the dimensions of the housing were restricted to the dimension of the table. The dimensions of the multi-touch housing were calculated via CAD to fit the table surface. The tangible table was constructed in a lab and the multi-touch table was built in the Bartlett School of Architecture workshop.
Both housing constructions made allowances for a workspace around the edges of the table. For the tangible table, we subtracted a few inches from the length and width of the table and made this the length and width of the housing. In the tangible table example, this provided the children with an area for placing the tangible artefacts when not in use. With the multi-touch table, we constructed a wide bezel on all sides of the table to allow participants to rest their elbows without triggering false touches with their forearms [13]. This bezel was also used to encase the projector and other devices, to eliminate obstrution of reflected light and to keep the projetion surface free of shadows.

We left a $2 \mathrm{~mm}$ gap between the tangible table surface and the housing so that if the table surface was knocked the projection underneath was not affected (Figure 1). This was an important design decision - we knew that our user group was children, and children can be very active! We needed interaction to continue despite possible movement to the table. The multitouch housing was constructed as part of the table rather than as two separate parts.

To keep the tangible table portable, we constructed the housing out of chipboard and pine, so that despite measuring $1240 \times 600 \times 695 \mathrm{~mm}$ it was very lightweight. Since the multitouch table was standing height and standing height tables are often used as leaning posts, we built an internal structure from square section steel beams and attached MDF paneling to sides and solid wood beams to the top perimeter of this structure. Heavy-duty casters allowed this considerable weight to be moved easily.

After placing the projector, IR LEDs and camera inside the tangible housing, we realized that the projector ran extremely hot and would automatically shut down after 5-10 minutes. Initially, we used a small office fan, and cut two holes in one side of the table to provide adequate ventilation (we later replaced the office fan with three built in computer fans). A simple cooling system was designed for the multi-touch table, to draw cold air in from the bottom of the table and push hot air (particularly from the projector) out of vents surrounding the surface.

\subsection{Illumination}

In both tabletops, extensive IR illumination was required to cover the large surface areas. Both tabletops tested several IR LEDs. With the tangible tabletop, we tested for brightness and viewing angle, first constructing an array of eight LEDs in parallel (LD271-950nm, 50 beam angle; YH70-940nm, 40 beam angle) and changing the distance between the LEDs in the array as well as the distance between the LEDs and the camera.

To illuminate the table surface, both studies then tested a wideangle CCTV night-vision illuminator which contained 48 highpowered LEDs $\left(850 \mathrm{~nm}, 140^{\circ}\right.$ beam angle). To test the tangible tabletop, we placed this in one corner of the housing and tested whether or not we could see a fiducial through the frosted surface of the table. Indeed we could; however, the illumination was much narrower than specified and it could only illuminate one corner of the table. We made several attempts to diffuse the light with white card, aluminum, foam packaging, and then tried the same type of illuminator with the LEDs turned at a slight inward angle but again this did not prove useful.

Likewise, with the multi-touch table, a single night-vision unit could not illuminate the entire surface directly. To resolve this issue, we purchased two similar units each using 48 of the same LEDs with a $60^{\circ}$ beam angle. We arranged the units in a way to provide both sufficient and consistent illumination across the surface (Figure 3 ). 


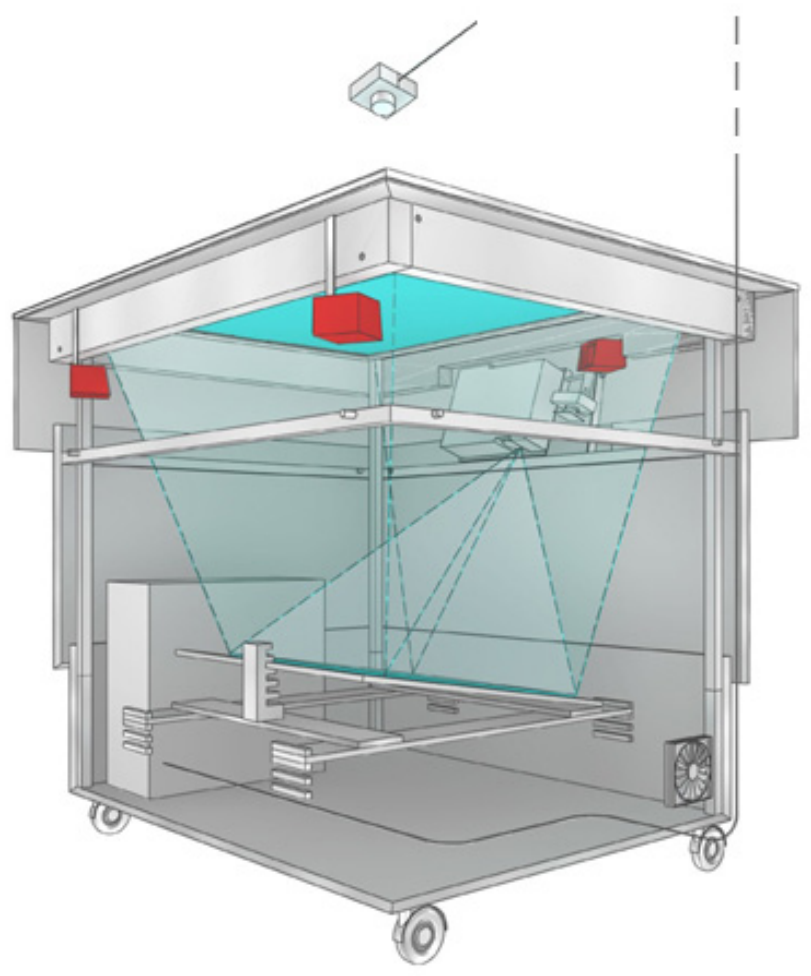

Figure 3: Multi-touch table schematic. Red boxes are IR illumination sources.

For the tangible table, our final solution was to build 8 boards of SFH485 (880nm) LEDs: 4 boards with 144 LEDs and 4 corner boards with 18 LEDs on each board, for a total of 648 LEDs. We placed these on the floor of the table around each side of the camera and in each corner of the table on a slight angle facing the surface (Figure 4). Once the LEDs were in place, the interior of the tangible table was painted black to decrease reflection.

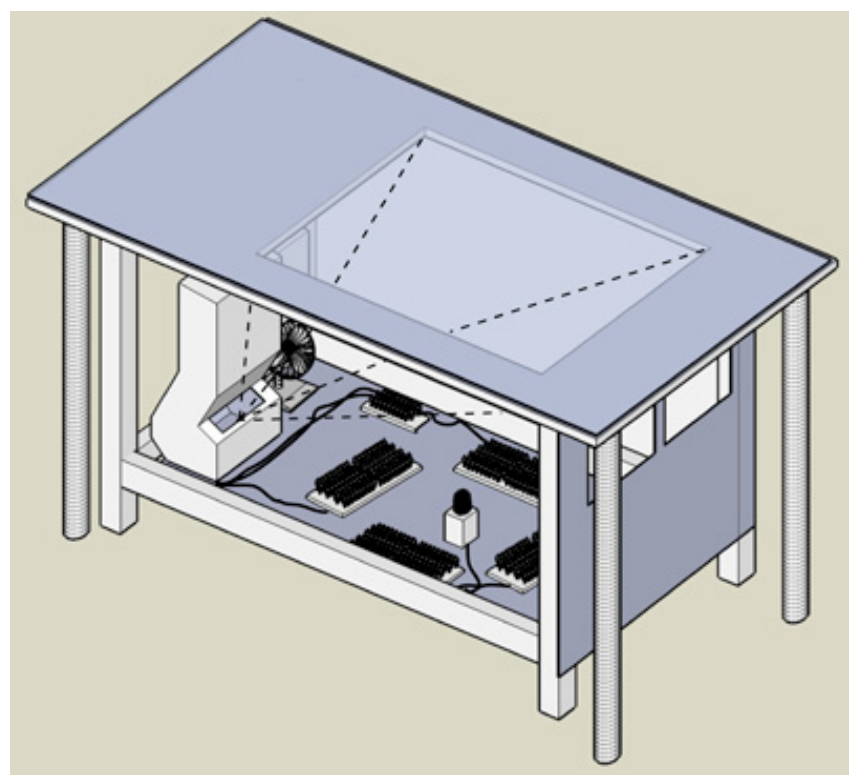

Figure 4: Tangible table schematic.
We investigated using different internal surfaces to aid diffusion/reflection (aluminium foil, Fresnel lenses) on the multi-touch surface, but like in the tangible table they made little difference above and beyond simply painting the interior white. To improve illumination we placed white card at angles toward the surface such that reflected light brightened the edges of the surface where required. This technique worked well with our configuration; however, it may not work as well in other configurations (Figure 3).

\subsection{Tracking interaction}

The two tabletops are somewhat different in their interaction tracking requirements. The tangible tabletop required recognition of different physical objects whereas the multitouch table required identification of individual users. Many different types of materials were tested for the design the physical artefacts for the tangible tabletop (Figure 5).
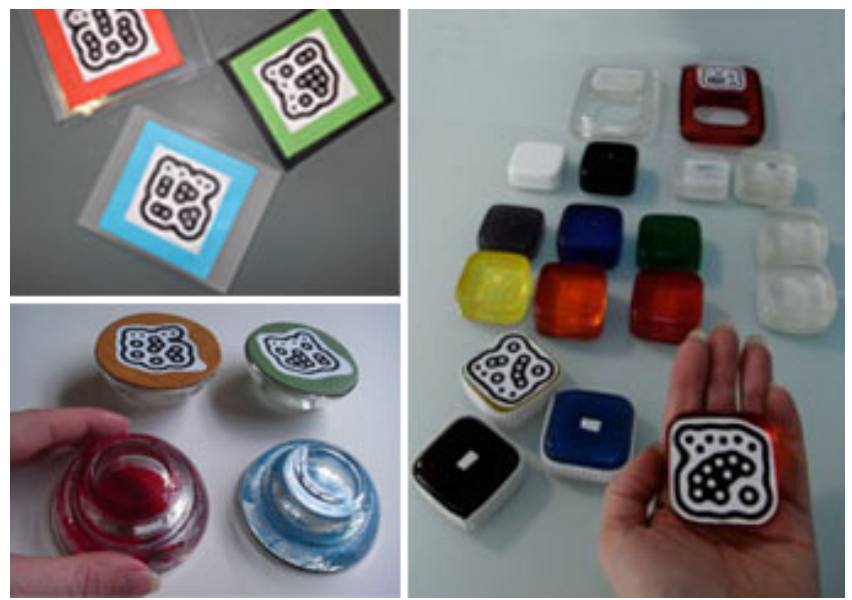

Figure 5. Iterative development of tangible artefacts with printed fiducials. Perspex and paper (top left); glass and leather (bottom left); crystal resin (right).

First, we used colored paper glued and then Perspex blocks but neither was the appropriate size and both had sharp edges. We then used glass tea light candleholders and leather. While this design was visually appealing, it wasn't very practical - the leather curled up at the edges and using glass presented a danger. Next, we tried Polymorph material, which molded easily but we could not create clean edges. Finally, we used Gedeo crystal resin, which imitates glass paste and dries transparent. This worked well and so we made 20 lightweight artefacts and painted each one with glass paint.

For the multi-touch table, we needed to identify who had touched the table as well as where they had touched the table. Since vision-based multi-touch surfaces cannot identify individual users solely based on their fingertips we introduced a second tracking method. We investigated many different systems, including colour-based, motion-based and featurebased tracking coupled with different types of references and targets such as rings, retro-reflective/neon tapes, and patterned gloves. However, for simplicity, we decided to use a markerbased tracking system as is often used for augmented reality. Whilst this does restrict the range of hand motion available to participants, this kind of tracking does not suffer from the initialization problems of colour-based tracking and is sufficiently robust to scale and rotation. A large marker $(100 \times 100 \mathrm{~mm})$ was placed on the back of the participant's hands without restricting finger movement (Figure 6). 


\section{J. Sheridan et al.}

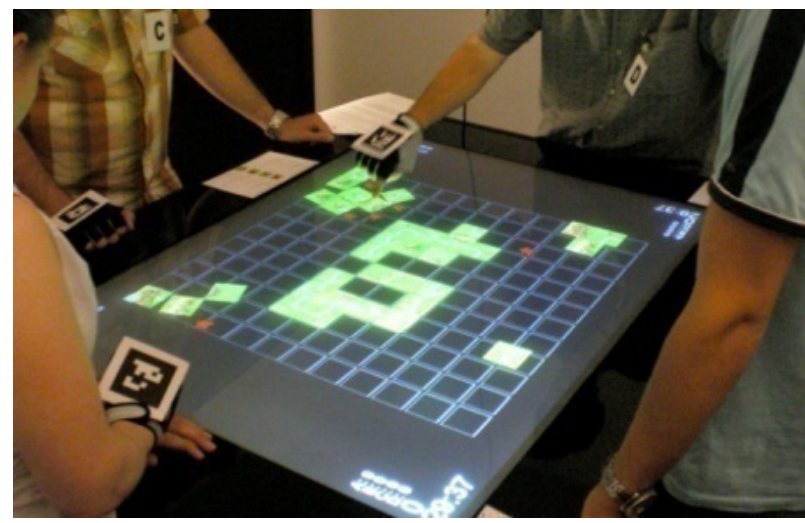

Figure 6. Interacting with multi-touch tabletop through glove-based marker tracking.

\subsection{Software}

Both tabletops made use of open source software to generate TUIO [5] events. The tangible tabletop used reacTIVision for marker tracking and Processing for the graphical interface. The multi-touch table used the TouchLib library for finger tracking, JOGL for the interface and the Phys2D library for tile interactions.

\section{LESSONS LEARNED}

Both teams of developers had to consider many trade-offs when designing our interactive tabletops. The three major dimensions that affected our outcomes were cost, time and experience. We explain these in more detail in this section.

Reducing the table height to suit children affected the distance between our projector/camera and surface which in turn affected the size of our interaction surface. This meant purchasing an expensive ultra short throw projector but ultimately this improved our portability - we reduced the need to calibrate a mirror as well as decreased our table size. The multi-touch surface was designed with adult use in mind so the height was increased. Since the multi-touch surface was built with a smaller budget, we re-used lab materials, such as the projection surface, which then affected the kinds of materials that were used for the housing, and ultimately affected the weight and size of the table. A trade-off was made between size and portability: we forfeited being able to fit the table easily through doors for a large surface that could accommodate four adults. Whilst it was difficult to calculate an exact interior temperature without first building the housing and running all of the equipment, the multi-touch table integrated a cooling system into the design from the start.

Environmental lighting affected how many lumens our projectors needed to be. With the tangible tabletop, blacking out the room was not a possibility since our user group was children and this became a health and safety issue. Also, the type of projector, camera and surface affected how much IR illumination was needed. The way in which the two different designs recognised fiducials also determined how the surface was illuminated. In both cases, many more IR LEDs were needed than was originally thought. This was a critical component to the success of both table designs and required many hours of fine tuning, particularly with adjusting camera settings and image preprocessing steps.

With the tangible tabletop, we tested many different thicknesses and frosted surfaces and yet we found it difficult to make a decision without first having the appropriate camera, projector and IR illumination. However, we did find that the best surfaces are frosted on one side and clear on the other, and that thinner surfaces (under $10 \mathrm{~mm}$ ) reduce refraction effects and blurring. For multi-touch finger input, the surface needs to diffuse light sufficiently to reduce potential false-touches. Another point to consider is that the location of the projector affects refraction but again, using a thinner surface reduces this problem. However, surfaces should not be so thin as to not be able to withstand the weight of the proposed interaction.

In both cases, camera parameters were adjusted through software which allowed us to fine-tune gain, exposure length, and shutter time. For the multi-touch table, we were able to match the camera's resolution and the size of the table to reach the desired precision. Likewise, the tangible table required a high-resolution camera with a wide-angle lens to see the largest interaction area possible. Since a smaller table will have a smaller interaction surface, a lower resolution camera could be used. Whilst the addition of a wide angle, fish-eye or zoom lens allows for greater flexibility in terms of camera placement - in particular, the distance between camera and projection surface barrel distortion can be a problem. In the tangible interaction example, barrel distortion was corrected using reacTIVision software. For cutting out visible light, IR longpass filters are an inexpensive option and are the only option when using a fisheye lens. IR bandpass filters matched to the wavelength of the IR illumination provided the best results.

\section{COMPARATIVE ANALYSIS}

A comparative analysis provides a frame of reference from which to compare and contrast our two tables. In a comparative analysis, the results are subjective and will vary depending on the perspective and experience of the individual(s) involved.

We approached our analysis from a Western perspective as well as from two teams of people who have hobbyist/professional experience (the division between hobbyist/professional is unclear at this time since the pool of people building interactive tables is relatively small). For our analysis, we used the three dimensions that emerged in our lessons learned (Section 5): cost, time and experience. We describe these as:

- Cost: Financial consideration of materials, labour and space.

- Time: Amount of time needed to complete the task. This dimension considers such things as how long it takes to source and acquire materials.

- Experience: Type of experience required to complete the task.

We further divided our dimensions into a rating scale of 1 to 5 (Table 1). The two teams of developers were then asked to rate several elements: concept, environment, computer, projector, camera, lens/filter, surface, housing, cooling, illumination, tracking, and software. We then mapped the ratings onto a radar chart (Figure 7 and Figure 8).

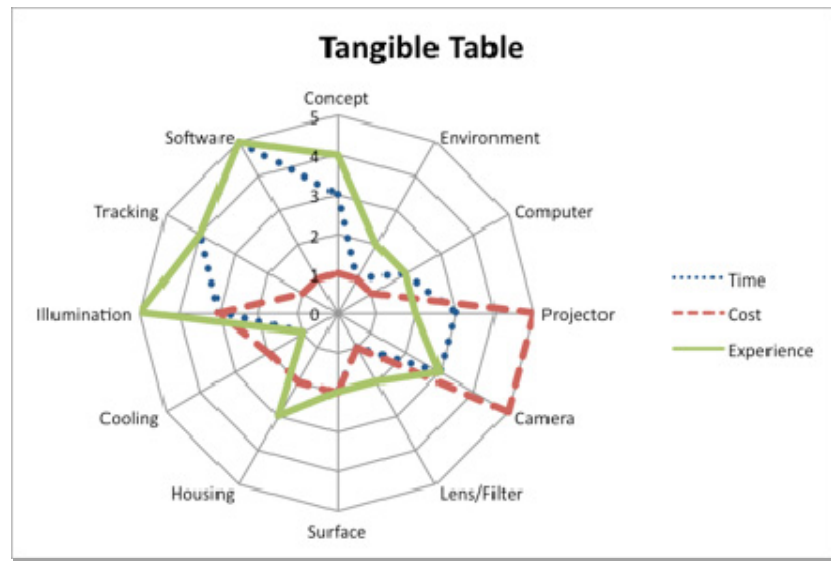

Figure 7. A radar chart of cost, time and experience in relation to the tangible table. 
DIY Design Process for Interactive Surfaces

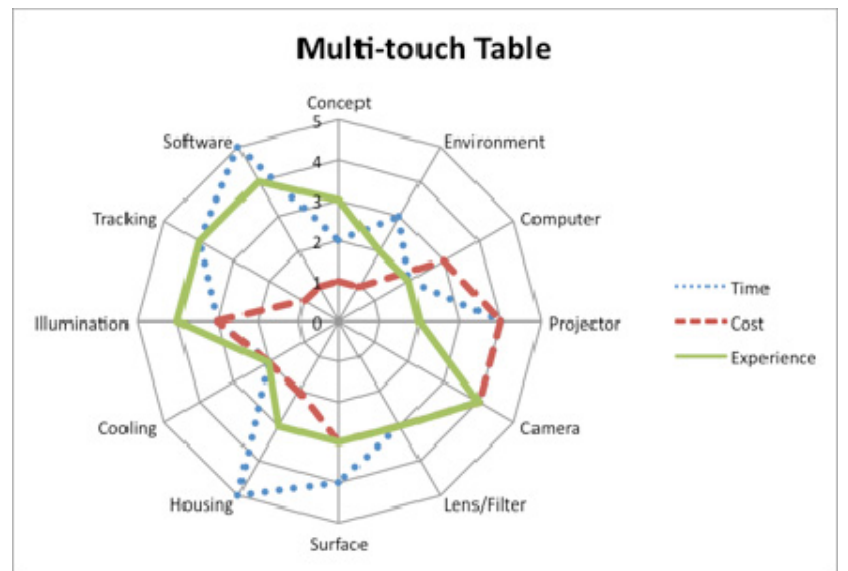

Figure 8. A radar chart of cost, time and experience in relation to the multi-touch table.

On initial inspection, it is clear that different patterns emerged from the two different figures. First, we will examine each of the dimensions in more detail and then we will consider how time, cost and experience compare across the two approaches.

\subsection{Time}

The time spent on various elements of both tables is shown in Figure 9. The multi-touch table's housing took much longer to build than the tangible table as both the surface and the housing were bespoke whereas part of the tangible table surface was purchased off-the-shelf.

The housing for the multi-touch table was built in a machine shop to precise measurements from CAD, whereas the tangible table was built to a rough sketch, the surface was purchased offthe-shelf and the housing was built in-house with inexpensive materials from a DIY shop. Constructing the table in-house and purchasing off-the-shelf or recycled components can save a significant amount of building time. Interesting is the fact that although a small amount of time was spent on configuring the computer, the biggest amount of time (for both approaches) was spent on developing software applications. This suggests (particularly with the tangible table) that the physical build of the tables and the computer equipment is less of an issue than developing software applications that truly benefit from the opportunities presented by interactive tables.

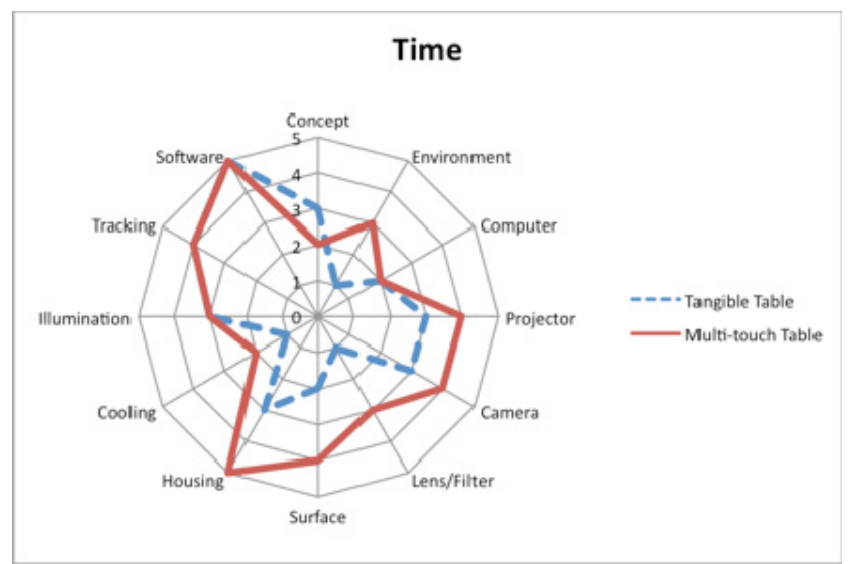

Figure 9. Radar chart of time spent on tables.

\subsection{Cost}

The amount of financial investment spent on the various elements is shown in Figure 10. Both of the table designs were vision-based and the figure shows that there is a clear trend towards the projector and camera, as these are the most critical elements of a vision-based system. Likewise, the multi-touch table pushes out towards the surface and lens/filter. Again, as these are critical elements of the system, it is necessary that a significant investment be made on these elements.

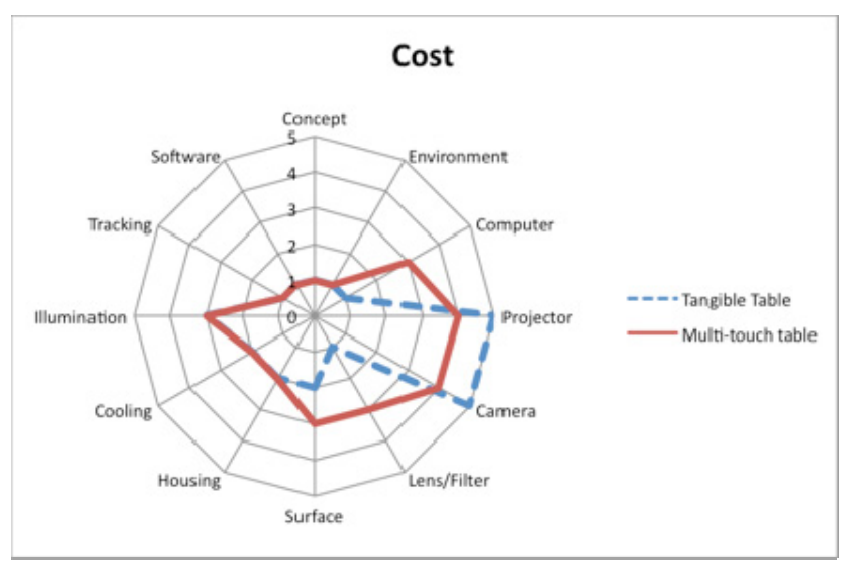

Figure 10. Radar chart of cost spent on tables.

With the tangible table there is a notable difference in terms of the projector and camera. This fact can be attributed to the inexpensive surface: since the tangible table used an inexpensive surface, a better projector and camera was needed. Additionally, even though both illumination approaches were quite different, the cost of the illumination equipment was approximately the same for both approaches.

\subsection{Experience}

We see in the experience ratings chart (Figure 11) that at most, there is only one point difference in any element between the two approaches. This suggests that regardless of which approach is used to build the table, prior hobbyist/professional experience has a particular learning curve.

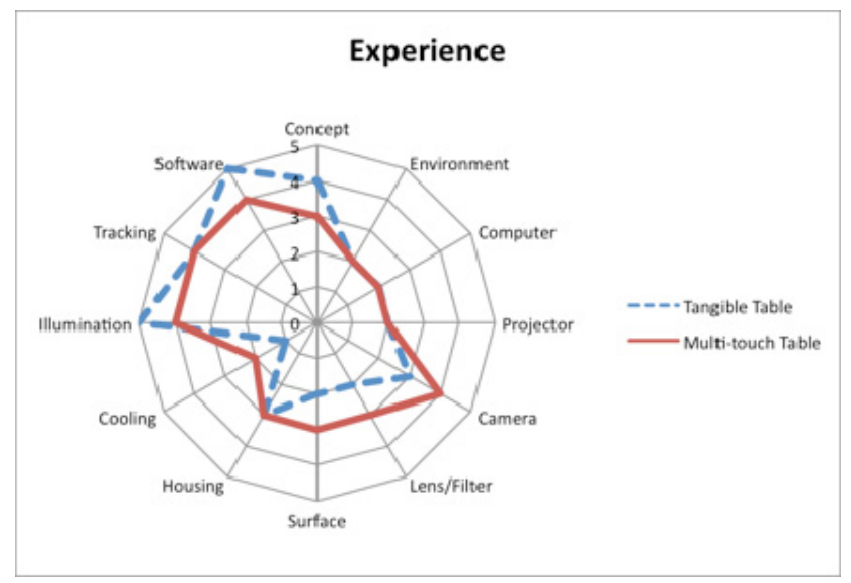

Figure 11. Radar chart of experience spent on tables.

The graph suggests where the learning curve is for hobbyist/professionals. In our two cases studies, the developers were people who had a significant amount of experience in building and developing software yet there is a notable trend towards illumination, tracking and software. This suggests that whilst the physical build might be fairly straight forward, there is a large learning curve in terms of software application development for those wishing to develop interactive tables 


\section{J. Sheridan et al.}

regardless of experience. For those who were not professionals or software-skilled hobbyists, the learning curve would be even more significant. This is most likely due to the fact that there are relatively few people developing interactive tables.

Where there is less significance, this suggests that hobbyist/professional experience with these elements (such as the camera and computer) is commonplace and these elements are most likely considered 'plug and play'. For hobbyist/professionals, these elements do not require much modification or specialist knowledge - they are elements which are part of the professional's previous experience and therefore in their repertoire of skills.

\section{DIY DESIGN PROCESS}

Reflecting on the experience of building the two tabletops described in this paper, we propose the following so-called DIY Design Process for interactive surfaces which is based our understanding of the tradeoffs involved in different options detailed in the radar figures of the previous section. This process has three steps:

(i) Identify the specific requirements of the physical environment, the corporeal abilities of the users, and the tangible features of the application to be developed.

(ii) Assess the expertise available to the development team in terms of software, electronics and carpentry.

(iii) Find the most fitting tradeoff suggested by the radar chart provided here.

When considering the physical setting for tabletop use the primary consideration is form factor. This defines the situation and the ambient lighting, which will in turn define the overall size limits of the surface and the type of IR illumination required for consistent operation. Additional considerations include access to power and related health and safety requirements, especially taking into account the potential heat dissipation due to table operation. Last but not least, consider whether the physical location allows for unattended operation or operation from non-specialist personnel. When considering users, important aspects are the age range and the resulting size limitations, any special needs they may have as well as their potential ability to manipulate objects of different sizes and form factors. This relates directly to specific application requirements, for example: the minimum and maximum interaction area that can provide adequate support for interaction and for locating objects without cluttering the surface; whether object or finger tracking are most appropriate modalities; and, last but certainly not least, whether users should be uniquely and consistently identifiable through a single ID.

On the other side of the formula is that it is critical to assess the particular skills and capabilities available. For example, whether it is possible to construct electronic circuits to control and provide more powerful or configurable lighting or flexible ventilation. Second, it is critical to match the software development capabilities of the team to the task. For example, considering which software components to reuse from those widely available and which ones to develop in house, or indeed what should be the most appropriate language for developing applications. Last but not least, consider the capability of the team to build the physical table itself and access to specialized equipment and materials, for example, laser cutters and 3D printers for the construction of complex artifacts.

The last step requires a quick search of the design space for a good compromise between requirements and capabilities. In this task the radar charts should be of great value as they summarize in a simple, usable and intuitive way the main issues to consider. This is not to say that they exhaust the design space and in some cases it may be possible to come up with solutions not covered here. For example, in cases where advanced software expertise is available and there is need for a very large interaction area with a reduced cost, one solution might be to multiplex several cameras into a tile pattern, which would require integration through software translation.

\section{SUMMARY}

Our paper charts the design, development and analysis of two interactive tabletops. The three key dimensions of time, cost and experience, which grew out of our lessons learned, inform our comparative analysis. We introduce the DIY Design Process for interactive surfaces and identify three key steps that designers should consider when building an interactive table. Our intention is to provide detailed documentation of our process to reduce the learning curve for other designers who want to develop their own interactive surface.

\section{FUTURE WORK}

The tabletops discussed in this paper have been developed within the context of extensive investigations into the use of interactive surfaces. They represent the first generation of such surfaces developed by the respective groups and building on the lessons learnt further iterations and next generation prototypes are being fabricated. Additionally, the tables will be shown and evaluated by a public audience (such as during the 'Surface Tension' event at the Science Museum's Dana Centre (UK) [20]. As such, we intend to continuously develop and refine our proposed DIY design process to incorporate additional technologies and related trade-offs.

\section{ACKNOWLEDGMENTS}

The tangible table is funded by the EPSRC grant number EP/F018436. Thanks to Sara Price (PI, DeTaLe project at the LKL), Taciana Pontual-Falcao (LKL), Demetrios Airantzis (Birkbeck) and Stephen Chapman (IoE). The multi-touch table is funded by the EngD VEIV Centre at UCL. Thanks to Professor Stephen Gage for advice on conceptual design, Artemis Skarlatidou and Patrick Weber for experiment design contribution and The Bartlett School of Architecture for extensive support on the construction of the multi-touch table.

\section{REFERENCES}

[1] Wellner, P. Interacting with Paper on the DigitalDesk. Commun. of the ACM 36, (1993) 87-96.

[2] Dietz, P.H. and Leigh, D.L. DiamondTouch: A Multi-User Touch Technology. Proc of UIST, (November 2001), ACM Press, 219-226.

[3] Ullmer B. and Ishii, H. The metaDESK: models and prototypes for tangible user interfaces. Proc. of UIST'97, (Banff Canada, 1997), ACM Press, 223-232.

[4] A. D. Wilson, "Playanywhere: a compact interactive tabletop projection-vision system," in UIST '05: Proceedings of the 18th annual ACM symposium on User interface software and technology. New York, NY, USA: ACM, 2005, pp. 83-92.

[5] http://www.natural-ui.com/aboutnui/whatwedo.html [Last accessed: May 14, 2009].

[6] Kaltenbrunner, M. and Bencina, R. reacTIVision: A Computer-Vision Framework for Table-Based Tangible Interaction. Proc of TEI 07 (Baton Rouge LO, February 2007), ACM Press, 69-74.

[7] Kaltenbrunner, M., Bovermann, T., Bencina R. and Costanza E. TUIO: A protocol for table-top tangible user interfaces. Proc. of the 6th Int'l Workshop on Gesture in 
Human-Computer Interaction and Simulation, (Vannes France, May 2005).

[8] Jordà, S. On stage: the Reactable and other Musical Tangibles go real, International Journal of Arts and Technology. Special Issue on Tangible and Embedded Interaction. Volume 1(3-4) (January 2009), pp. 268-287.

[9] Designing Tangibles for Learning project (DeTaLe): http://www.lkl.ac.uk/research/tangibles/ [Last accessed: May 9, 2009].

[10] Price, S. A representation approach to conceptualizing tangible learning environments. In Proceedings of the 2 nd International Conference on Tangible and Embedded Interaction 2008 (TEI'08), Bonn, Germany, February 1820, 2008, pp. 151-158.

[11] Price, S., Sheridan, J.G., Pontual-Falcão, T. and Roussos, G. Towards a Framework for Investigating Tangible Environments for Learning. International Journal of Arts and Technology. Special Issue on Tangible and Embedded Interaction. Vol.1, Nos. 3/4, (2008), 351-368.

[12] Wrede, K.J. Carcassonne. Rio Grande Games, 2001. http://www.riograndegames.com [Last accessed: May 9, 2009].

[13] Appelcline, S. Anatomy of a Game: Carcassonne, Parts 1 to 3. (March 30 ${ }^{\text {th }} 2006$ ) Gone Gaming: http://boredgamegeeks.blogspot.com/2006/03/anatomy-ofgame-carcassonne-part-one.html. [Last accessed: May 9, 2009].

[14] Price, S., Pontual-Falcão, T., Sheridan, J.G. and Roussos, $\mathrm{G}$. The effect of representation location on interaction in a tangible learning environment. In Proceedings of the 3rd International Conference on Tangible and Embedded Interaction, Cambridge, UK.

[15] Pontual Falcão, T., Price, S. What have you done! The role of 'interference' in tangible environments for supporting collaborative learning. In Proceedings of the 8th International Conference on Computer Supported Collaborative Learning, CSCL'09. June 2009, Rhodes, Greece.

[16] Jordà, S. Sonigraphical Instruments: From FMOL to the reacTable. Proc of the NIME 03 (Montreal, PQ, 2003), 7076.

[17] Ryall, K., Forlines, C., Shen, C., Morris, M.R., Everitt, K. Experiences with and Observations of Direct-Touch Tabletops. Proc. of Horizontal Interactive HumanComputer Systems (TABLETOP'06), (Adelaide Australia, January 2006), 89-96.

[18] Antle, A. The CTI framework: informing the design of tangible systems for children. Proc of TEI 07 (Baton Rouge LO, February 2007), ACM Press, 195-202.

[19] Wigdor, D., Penn, G., Ryall, K., Esenther, A. and Shen, C. Living with a Tabletop: Analysis and Observations of Long Term Office Use of a Multi-Touch Table. Proc. of Horizontal Interactive Human-Computer Systems (TABLETOP'07), (Newport RI, 2007) 60-67.

[20] Surface Tension. Science Museum's Dana Centre, June $23^{\text {rd }}, 2009$. http://www.danacentre.org.uk [Last accessed: June 09, 2009]. 